\title{
INTEGRATION AND EXPRESSION OF MARKER GENES IN CHICKEN EMBRYOS WITH THE USE OF RETROVIRAL VECTOR
}

\author{
N.A. Volkova', L.A. Volkova ${ }^{1}$, I.K. Fomin', N.A. Zinovieval, \\ L.Sh. Gorelik ${ }^{2}$, N.S. Lotsmanova ${ }^{3}$ \\ ${ }^{1}$ All-Russia Research and Development Institute for Livestock Husbandry, RAAS, Dubrovitsy settlement 142132, Moscow province, Russia \\ e-mail:natavolkova@inbox.ru \\ ${ }^{2}$ Ural State Academy of Veterinary Medicine, Troitsk 457100, Chelyabinsk province, Russia \\ e-mail:natavolkova@inbox.ru \\ ${ }^{3}$ Base Medical College ${ }^{1} 15$ of Moscow Healthcare Department, Moscow 109263, Russia \\ e-mail:natavolkova@inbox.ru
}

Received January 19, 2013

S u m m a ry

The efficiency was investigated of transfer of exogenous DNA to chicken embryos in vivo with the use of retroviral vectors. The gene construction involves a marker gene GFT under control of promoter of yearly genes of human cytomegalovirus CMV IE (pLNCgfp) or promoter of Moloney leukemia virus Mo-MuLV (pLgfpSN). Two packing lines GP + envAM12 and PT67 were used for the delivery of retroviral vectors. It was established the high efficiency of embryo transformation with the use of pLNCgfp gene construction (up to $18.8 \%$ ). The expression of marker gene was demonstrated in cells of 5- and 15-day age chicken embryos.

Keywords: cell engineering, transgenesis, retrovirus vectors, chicken.

Biological bases in creation of transgenic living forms are an urgent task of modern science. Transgenesis is seen as essential part of future biotechnology involved in development of various fundamental and applied issues. One of such promising directions of biotechnology is production of transgenic chickens-bioreactors (1-6).

Significant physiological differences between birds and mammals suggest a clear advantage of birds as a productive platform for synthesis of recombinant proteins with a complex structure: birds are immunoresistant to potential therapeutic proteins (eg, human erythropoietin) the expression of which may adversely affect the health of transgenic mammals in commercial production of such substances. Along with it, using transgenic birds as a productive platform significantly reduces the cost of resulting product compared with variants of microbial fermentation Escherichia coli, yeast, or mammalian cell culture (7).

A conventional method of producing transgenic animals (microinjection of DNA into the pronucleus of zygote) is ineffective in poultry, which necessitates alternative approaches for the transfer of exogenous DNA, such as the use of genetic constructs based on recombinant retroviruses. Their capacity for target delivery of foreign genes in dividing cells is quite relevant for transformation of embryonic cells in poultry, in particular chickens, since a hatched chicken egg contains an embryo developed by the stage of 50-60 thousand cells.

In this regard, the purpose of this work was studying the efficiency of transfer of recombinant DNA into chicken embryos in vivo with the use of different expressing retroviral vectors, performed as a preliminary stage in development and optimization of the technology of producing transgenic poultry.

Technique. Two gene constructs were introduced into chicken embryos - pLgfpSN and pLNCgfp designed based on retroviral vectors pLXSN and pLNCX, respectively (8). The constructs contained the following cis-acting elements of retroviral genome: 5'- and 3'-LTR (U3-R-U5); tRNA primer binding site (PBS, site of initiation of minus-strand DNA synthesis); $\psi$-region and fragment of gag gene (sequences responsible for dimerization and packaging of viral RNA); sd (splice donor site); polypurine tract (PPT, site of initiation of plus-strand DNA synthesis). To ensure the constructs' capability for selective introduction into cells of the packaging line, there was integrated the gene for resistance to the chemical analog of neomycin G418 (neo); its transcription was controlled from either the retroviral LTR (pLNCgfp), or from the promotor of early virus genes SV40 (pLgfpSN). In pLgfpSN gene construct, the marker gene GFP was subject to the control of Mo-MuLV promoter, in pLNCgfp - the promoter of human cytomegalovirus immediate early genes (CMV-IE). The vectors were packed with two lines of packaging cells - GP+envAM12 and PT67 $(9,10)$. Both these lines were derived based on mouse NIH 3T3 fibroblasts and differed in type of the produced env-proteins responsible for recognition of surface receptors on target cells.

A source of viral gene constructs was a virus preparation with the titer $9 \frac{1}{2} 105 \mathrm{cfu} / \mathrm{ml}$. The constructs were injected into the dorsal aorta of 2,5-day-old chicken embryos ( 2 microliters per embryo) using capillary mikropipet Transferpetor ("Sigma”, USA). The efficiency of transformation by retroviral vectors was determined in 5- and 15-day-old embryos by the presence and expression of GFP gene. The presence of GFP gene was identified by PCR of DNA derived from embryos by high salt extraction (11). The expression of GFP gene in embryos was evaluated in cryostat tissue sections by the presence of specific fluorescence ("Nicon" microscope, Japan; filter 480-490 nm). The cryosections were prepared according to a conventional method (12).

Results. The efficiency of embryo transformation varied depending on the used genetic construct and the line of packaging cells. The minimal impact on embryogenesis of chickens was observed in variants with gene constructs introduced with rT67 packaging line: the development of embryos was observed in $70-73 \%$ cases. In variants when gene constructs were introduced in chicken embryos with GP+envAM12 packaging line, embryonic mortality was 3-13\% higher (Table 1). There were no significant differences in effects of the used gene constructs on chicken embryogenesis. 
1. Efficiency of transgenesis in chicken embryos injected in vivo with retroviral vectors pLNCgfp, pLgfpSN, and different lines of packaging cells

\begin{tabular}{|c|c|c|c|c|}
\hline \multirow{3}{*}{ Parameter } & \multicolumn{4}{|c|}{ Line of packaging cells } \\
\hline & \multicolumn{2}{|c|}{$\mathrm{GP}+$ envAM12 } & \multicolumn{2}{|c|}{ pT67 } \\
\hline & pLNCgfp & pLgfpSN & pLNCgfp & pLgfpSN \\
\hline \multicolumn{5}{|c|}{$5^{\text {th }}$ day of embryo development } \\
\hline Examined eggs, pcs. & 30 & 30 & 30 & 30 \\
\hline Outcome of developed embryos, $n(\%)$ & $20(67)$ & $17(57)$ & $21(70)$ & $22(73)$ \\
\hline Outcome of transgenic embryos, $n$ & 14 & 10 & 10 & 9 \\
\hline Frequency of integration, $\%$ & 70,0 & 58,9 & 47,7 & 40,9 \\
\hline Efficiency of transgenesis, \% & 46,7 & 33,4 & 33,4 & 30,0 \\
\hline \multicolumn{5}{|c|}{$15^{\text {th }}$ day of embryo development } \\
\hline Examined eggs, pcs. & 48 & 42 & 51 & 55 \\
\hline Outcome of developed embryos, $n(\%)$ & $14(29)$ & $10(24)$ & $20(39)$ & $24(44)$ \\
\hline Outcome of transgenic embryos, $n$ & 9 & 5 & 6 & 5 \\
\hline Frequency of integration, $\%$ & 64,3 & 50,0 & 30,0 & 20,9 \\
\hline Efficiency of transgenesis, $\%$ & 18,8 & 11,9 & 11,8 & 9,1 \\
\hline
\end{tabular}

High efficiency of genetic transformation of chicken embryos was provided by the genetic construct pLNCgfp: the proportion of transformed embryos relative to the total number of injected ones amounted $46,7 \%$. In the variant with the gene construct pLgfpSN the parameter was $13,3-37,6 \%$ lower and ranged from 9,1 to $33,4 \%$.

In these experiments, the use of GP+envAM12 packaging line resulted in 2,8-13,3\% higher frequency of integration of the recombinant DNA and higher efficiency of transgenesis compared with the line rT67.

Studying the efficiency of transfer of recombinant DNA in chicken embryos on the $5^{\text {th }}$ and $15^{\text {th }}$ day of development, it was revealed the decline in proportion of transgenic embryos with increasing time of incubation. Thus, for the gene construct pLNCgfp, the percentage of transgenic embryos on the $5^{\text {th }}$ day was $46,7 \%$, and on the $15^{\text {th }}$ day $-18,8 \%$ from the total number of treated embryos. A similar trend was found in variants with the gene construct $\mathrm{pLgfpSN}$ : from the $5^{\text {th }}$ to the $15^{\text {th }}$ day, this parameter decreased by $20,9-21,5 \%$ depending on the used line of packaging cells. Such decline in number of transgenic embryos with increasing time of incubation indicates the influence of transgene expression on embryogenesis.

Topographical analysis of integration patterns showed that the highest rate of transformed organs and tissues (42\%) was observed in cases of using the gene construct pLNCgfp and the packaging line GP+envAM12 (Table 2). Expression of the reporter protein was detected primarily in the liver, heart and muscle tissue. In the variant of the gene construct pLgfpSN and the packaging line rT67, the efficiency of transformation of organs and tissues was $11 \%$ lower and amounted $31 \%$.

\section{Topography of genetic transformation in 15-days-old chicken embryos injected in vivio with retroviral vectors $p L N C g f p, p L g f p S N$, and different lines of packaging cells}

\begin{tabular}{|c|c|c|c|c|}
\hline \multirow{3}{*}{ Parameter } & \multicolumn{4}{|c|}{ Line of packaging cells } \\
\hline & \multicolumn{2}{|c|}{ GP + envAM12 } & \multicolumn{2}{|c|}{ pT67 } \\
\hline & pLNCgfp & pLgfpSN & pLNCgfp & pLgfpSN \\
\hline Transgenic embryos, $n$ & 9 & 5 & 6 & 5 \\
\hline Investigated organs, $n$ & 5 & 5 & 5 & 5 \\
\hline Transformed organs on average over the group, $\%$ & 42 & 40 & 34 & 31 \\
\hline
\end{tabular}

The expression of GFP gene in organs and tissues of 5- and 15-day-old chicken embryos is shown in Figure (see insert).

Thus, experiments on the transfer of recombinant DNA into chicken embryos in vivo suggest good prospects in the use of retroviral vectors for genetic transformation of embryonic cells and obtaining transgenic poultry. High efficiency of transgenesis $(18,8 \%)$ was observed in the variant of using the gene construct pLNCgfp and the packaging cell line GP + envAM12.

\section{RERERENCES}

1. Kamihira M., Nishijima K., Iijima S. Transgenic birds for the production of recombinant proteins. Adv. Biochem. Eng. Biotechnol., $2004,91:$ 171-189.

2. Kamihira M., Ono K., Esaka K. et al. High-level expression of single-chain Fv-Fc fusion protein in serum and egg white of genetically manipulated chickens by using a retroviral vector. J. Virol., 2005, 79: 10864-10874.

3. Kiies W.A., Niemann H. The contribution of farm animals to human health. Trends Biotechnol., 2004, 22: $286-294$.

4. Rapp J.C., Harvey A.J., Speksnijder G.L. et al. Biologically active human interferon alpha-2b produced in the egg white of transgenic hens. Transgenic Res., 2003,

12: $569-575$.

5. Ernst L.K., Volkova N.A., Zinov'eva N.A. Sel'skokhozyaistvennaya Biologiya [Agricultural Biology], 2009, 2: 4-9.

6. Scott B.B., Velho T.A., Sim S., Lois C. Applications of avian transgenesis. ILARJ., 2010, 51(4): 353-361.

7. Ivarie R. Avian transgenesis: progress towards the promise. Trends Biotechnol., 2003, 21: 14-19.

8. Miller A.D., Rosman G.J. Improved retroviral vectors for gene transfer and expression. Biotechniques, 1989, 7: 980-990.

9. Markowitz D., Goff S., Bank A. Construction and use og a safe and efficient amphotropic packaging cell line. Virology, 1988, 167: 400-406.

10. Miller A.D., Chen F. Retrovirus packaging cells based on 10A1 murine leukemia virus for production of vectors that use multiple receptors for cell entry. Virology, 1996, 70: 5564-5571.

11. Zinov'eva N.A., Popov A.N., Ernst L.K. et al. Metodicheskie rekomendatsii po ispol'zovaniyu metoda polimeraznoi tsepnoi reaktsii v zhivotnovodstve [Guidelines for PCR in Animal Husbandry]. Dubrovitsy, 1998.

12. Mikroskopicheskaya tekhnika: rukovodstvo /Pod red. D.S. Sarkizova, Yu.P. Perova [Microscopy Technique: Guidelines. D.S. Sarkizova, Yu.P. Perova (eds.)]. Moskva, 1996. 